\title{
Mesa-size-dependent color contrast in flip-chip blue/green two-color InGaN/GaN multi-quantum-well micro-light-emitting diodes
}

\author{
Horng-Shyang Chen, Dong-Ming Yeh, Chih-Feng Lu, Chi-Feng Huang, \\ Yen-Cheng Lu, Cheng-Yen Chen, Jian-Jang Huang, and C. C. Yang ${ }^{\text {a) }}$ \\ Graduate Institute of Electro-Optical Engineering, National Taiwan University, Taipei, 10617 Taiwan, \\ Republic of China and Department of Electrical Engineering, National Taiwan University, Taipei, 10617 \\ Taiwan, Republic of China
}

(Received 5 June 2006; accepted 6 July 2006; published online 28 August 2006)

\begin{abstract}
The authors fabricate blue/green two-wavelength, InGaN/GaN quantum-well (QW), flip-chip micro-light-emitting diodes ( $\mu$-LEDs) of different mesa sizes by stacking QWs of different indium contents. It is found that the blue/green contrast ratio of such a $\mu$-LED increases with the mesa size. The relatively stronger blue intensity in a device of a larger mesa area is due to its higher operation junction temperature such that hole migration can be enhanced through thermally exciting holes to escape from the QW (green emitting) closest to the $p$-type layer and to be captured by the neighboring QWs (blue emitting). The higher junction temperature in such a $\mu$-LED of a larger mesa area is due to its smaller ratio of the sidewall surface area over the active volume, leading to the less effective sidewall heat radiation and light extraction. (C) 2006 American Institute of Physics.

[DOI: $10.1063 / 1.2339034]$
\end{abstract}

The development of white-light light-emitting diode (LED) is quite promising for significantly saving the global energy. InGaN/GaN-based LED is expected to be an efficient, reliable, cost-effective, versatile geometry for the applications to the color display and lighting. Currently, the development of white-light LED relies on the use of phosphors for converting blue or UV photons, generated by a single-color InGaN/GaN-based LED, into longerwavelength complementary colors. However, the use of phosphors leads to the disadvantages of the Stokes-wave energy loss, low reliability, low color rendering index, and patent control. The use of InGaN/GaN compounds for more than one-color emission can lead to a more flexible control of color mixing in a more efficient and reliable LED. This development direction is particularly interesting because of the recent progresses of $\mathrm{InGaN} / \mathrm{GaN}$ quantum-well $(\mathrm{QW})$ growth for long-wavelength visible emission. ${ }^{1-4}$ By stacking such QWs of different indium contents and/or thicknesses, emissions of various visible wavelengths can be obtained. ${ }^{2-6}$ Based on this concept, an efficient single-chip allInGaN/GaN white-light LED can be implemented.

However, the aforementioned QW stacking for controllable multicolor emission faces the following problems: First, the strain condition of such a QW will lead to the influence of the earlier-grown QW on the subsequently grown ones, resulting in the difficulty of controlling the designated emission wavelength. ${ }^{7}$ Second, the short migration distance of holes in an InGaN compound implies the dominance of emission from the QW closest to the $p$-type layer in a LED structure. ${ }^{8-10}$ This factor makes the color contrast control of a multicolor LED difficult. In this letter, we demonstrate the variation of color contrast of a flip-chip blue/ green two-color micro-LED ( $\mu$-LED) of stacking two different $\mathrm{InGaN} / \mathrm{GaN}$ QWs by controlling the mesa size. Such LEDs have great potential for microcolor display. ${ }^{11-15}$ In our

\footnotetext{
a) Author to whom correspondence should be addressed; FAX: 886-2-
} 23652637; electronic mail: ccy@cc.ee.ntu.edu.tw devices, the $p$-type contacts cover the whole mesa areas. With different mesa or $p$-type contact areas in the $\mu$-LEDs, different thermal effects lead to different junction temperatures. In a device with a higher junction temperature, holes have a higher probability of thermal escape from the QW closest to the $p$-type GaN layer such that holes can reach the next QWs for the emission of another color.

The epistructure of the blue/green two-wavelength LED was grown with a metal-organic chemical vapor deposition reactor under the following growth conditions: After the growth of a $25 \mathrm{~nm}$ nucleation layer at $535{ }^{\circ} \mathrm{C}$, a $2 \mu \mathrm{m}$ $n$-GaN of $5 \times 10^{18} \mathrm{~cm}^{-3}$ in silicon-doping concentration was deposited at $1070{ }^{\circ} \mathrm{C}$. Then, two QW growth conditions were used: (1) the growth of green-emitting QW with temperature at $690{ }^{\circ} \mathrm{C}$, wafer carrier rotation at $750 \mathrm{rpm}$, and gas flow rates at 3000 SCCM (SCCM denotes cubic centimeter per minute at STP) for $\mathrm{N}_{2}$ and $3000 \mathrm{SCCM}$ for $\mathrm{NH}_{3}$, and (2) the growth of blue-emitting QW with temperature at $710^{\circ} \mathrm{C}$, wafer carrier rotation at $1500 \mathrm{rpm}$, and gas flow rates at $1000 \mathrm{SCCM}$ for $\mathrm{N}_{2}$ and $1500 \mathrm{SCCM}$ for $\mathrm{NH}_{3}$. With the different growth conditions above, the indium compositions are different, leading to the emissions of different colors. Purely blue and green LEDs were also fabricated based on the growth of five QWs under the same individual conditions. In the two-color QW sample, we arranged the QW sequence as green/blue/blue/green in the four-QW structure. All the well thicknesses are about $3 \mathrm{~nm}$. The lower GaN barrier of the bottom QW has the thickness of $16 \mathrm{~nm}$ and was grown at $800{ }^{\circ} \mathrm{C}$ with the silicon-doping concentration of $7 \times 10^{17} \mathrm{~cm}^{-3}$. Among the rest four barrier layers, the first two (from the top) have the thickness of $6 \mathrm{~nm}$ and the next two have the thickness of $16 \mathrm{~nm}$. In the deposition of a $6 \mathrm{~nm}$ barrier layer, after the growth of an $\sim 2 \mathrm{~nm} \mathrm{GaN}$ cap layer at the same temperature as that for the last well layer, the growth was interrupted. During the interruption, the wafer temperature was ramped to $800{ }^{\circ} \mathrm{C}$ and $\mathrm{H}_{2}$ of $500 \mathrm{SCCM}$ was added to the growth chamber. ${ }^{16}$ After the interruption, a $4 \mathrm{~nm} \mathrm{GaN} \mathrm{layer} \mathrm{was} \mathrm{grown} \mathrm{to} \mathrm{form} \mathrm{a} 6 \mathrm{~nm}$ barrier. The 


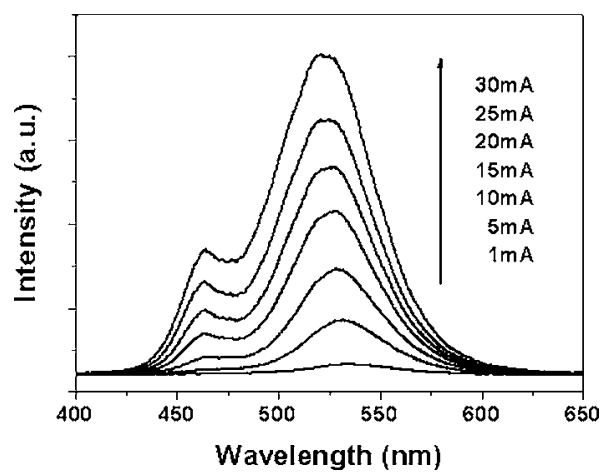

FIG. 1. Output spectra of a standard blue/green two-wavelength LED (sample A) at several injection current levels.

procedures for depositing a $16 \mathrm{~nm}$ barrier were the same as those for a $6 \mathrm{~nm}$ barrier except that a $14 \mathrm{~nm}$ GaN layer was grown at $800{ }^{\circ} \mathrm{C}$ after the growth interruption. The smaller barrier thickness close to the $p$-type layer was designed to enhance the hole trapping into the deeper QWs. After the growth of the four QWs, a $20 \mathrm{~nm} p-\mathrm{Al}_{0.2} \mathrm{Ga}_{0.8} \mathrm{~N}$ layer, followed by a $120 \mathrm{~nm} p$-GaN layer (both grown at $930{ }^{\circ} \mathrm{C}$ ), was deposited. LEDs based on this QW epistructure were then fabricated with the standard procedure. Ni $(15 \mathrm{~nm}) / \mathrm{Au}$ $(150 \mathrm{~nm})$ were used for the $p$-type Ohmic contacts. Also, Ti $(15 \mathrm{~nm}) / \mathrm{Al}(75 \mathrm{~nm}) / \mathrm{Ti}(15 \mathrm{~nm}) / \mathrm{Au}(150 \mathrm{~nm})$ were used for the $n$-type Ohmic contacts. Four device samples were fabricated for comparison. Sample A is a standard topemission LED with the mesa size of $300 \times 300 \mu \mathrm{m}^{2}$ and the $p$-type Ohmic contact size of $40 \times 40 \mu \mathrm{m}^{2}$. Thin metal layers of $\mathrm{Ni}(5 \mathrm{~nm}) / \mathrm{Au}(5 \mathrm{~nm})$ were coated to cover the whole top surface of the mesa for enhancing current spreading in the device. The other three device samples are flip-chip $\mu$-LEDs with the mesa areas of $10 \times 10$ (sample B), $20 \times 20$ (sample C), and $40 \times 40 \mu \mathrm{m}^{2}$ (sample D). The $p$-type contacts cover the whole mesa areas in the three flip-chip $\mu$-LEDs.

Figure 1 shows the LED output spectra of sample A at several normal-operation injection current levels. One can see that at the low current levels, almost only the green emission peak can be observed. However, as the injection current increases, the blue emission peak becomes stronger and stronger. The dominance of green emission at the low current levels is simply because the QW closest to the $p$-type layer is designated for green emission and the hole mobility in the InGaN/GaN QW structure is much lower than that of electron. Therefore, most of the holes are trapped by the first QW for green emission at the low current levels. As the injection current increases, the extra holes after filling up the first QW can reach the second one for blue emission. In other words, the blue/green color contrast can be controlled by the injection current density. In the normal operation of a standard two-color LED of stacking different QWs, the emission color of the QW closest to the $p$-type layer is stronger than another color from the next QW unless the injection current density is high.

The injection current dependence of color contrast in a two-wavelength LED provides us with the clue of controlling the color contrast by changing the mesa size in a flipchip $\mu$-LED. Parts (a), (b), and (c) of Fig. 2 show the current density dependent output spectra of samples B, C, and D, respectively. In each individual sample, the blue-over-green contrast ratio increases with injection current density, similar Downloaded 04 Feb 2009 to 140.112.113.225. Redistribution subje
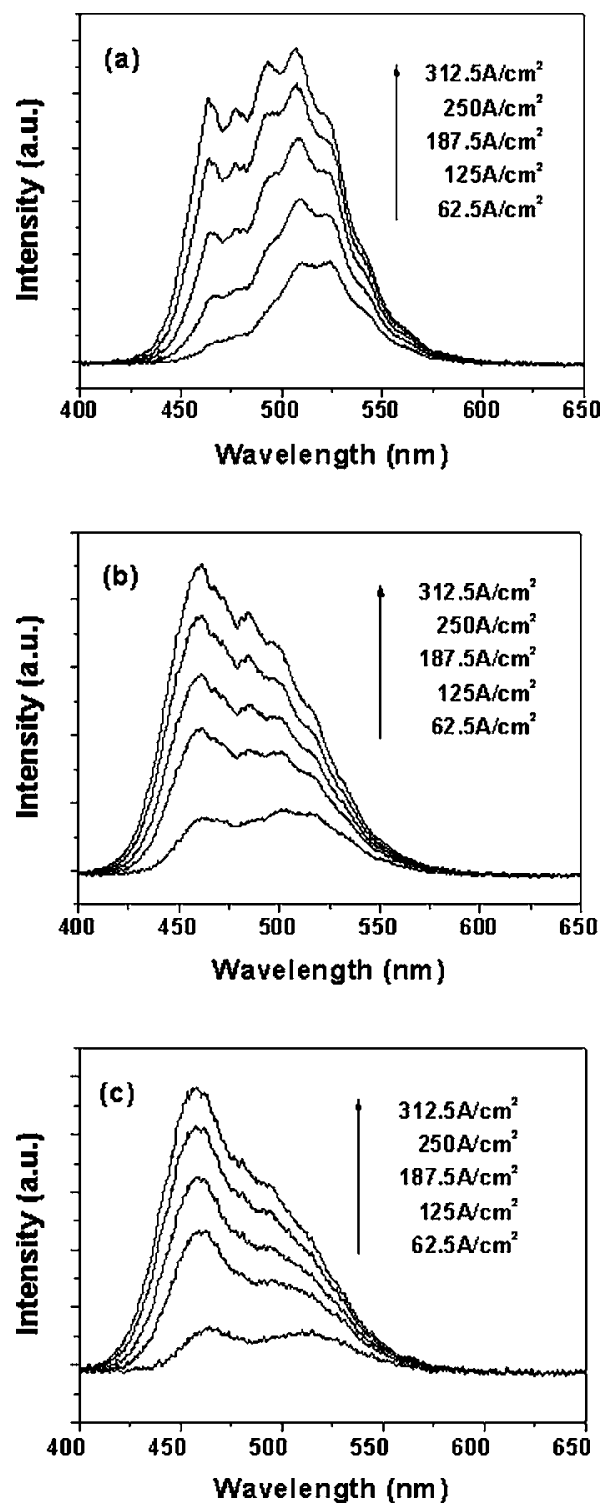

FIG. 2. Output spectra of flip-chip $\mu$-LEDs at several injection current densities with (a) a $10 \times 10 \mu \mathrm{m}^{2}$ mesa area (sample B), (b) a $20 \times 20 \mu \mathrm{m}^{2}$ mesa area (sample C), and (c) a $40 \times 40 \mu \mathrm{m}^{2}$ mesa area (sample D).

to the trend in Fig. 1. Meanwhile, among the three samples, this contrast ratio generally increases with the mesa size. In the device of $10 \times 10 \mu \mathrm{m}^{2}$ mesa area (sample B), over the current density range from 62.5 through $312.5 \mathrm{~A} / \mathrm{cm}^{2}$, the blue emission is always weaker than the green emission. However, in the device of $20 \times 20 \mu \mathrm{m}^{2}$ mesa area (sample $\mathrm{C})$, the blue-over-green contrast ratio increases from below unity to become larger than 1 as the current density increases over the same range. Then, in the device of $40 \times 40 \mu \mathrm{m}^{2}$ mesa area (sample D), the blue emission peak is always higher than the green one over the same current density range. Therefore, it is believed that a larger mesa or current injection area in the flip-chip $\mu$-LED leads to more hole trapping by the second (blue-emitting) QW.

Figure 3 shows the curves of the LED output power versus injection current density of the three $\mu$-LED samples. Here, plotting the three curves in the same figure means to compare their output saturation current densities. The saturation current densities reflect the relative junction temperatures among the three samples. One can see that a larger mesa area leads to a lower saturation current density. Figure to AIP license or copyright; see http://apl.aip.org/apl/copyright.jsp 


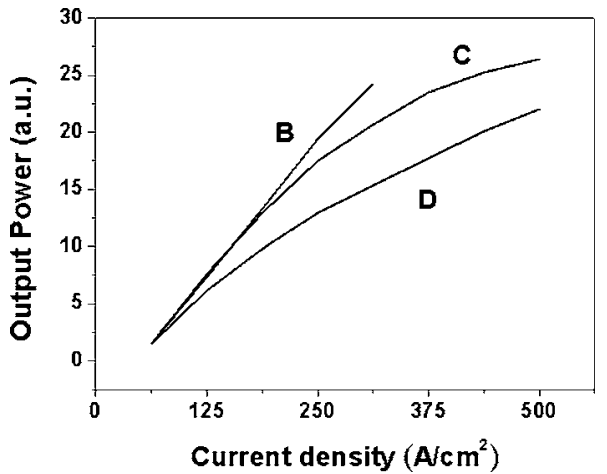

FIG. 3. Output power variations as functions of injection current density of the three flip-chip $\mu$-LEDs with different mesa areas.

4 shows the curves of current density versus applied voltage of samples B, C, and D. One can see that the turn-on voltages of the three samples are about the same at around $5 \mathrm{~V}$. However, their device conductivities are quite different. A larger mesa area leads to a larger conductivity.

The different spectral results among the three $\mu$-LEDs, as shown in Fig. 2, are believed to be due to their different junction temperatures. With a higher junction temperature, it is more likely for holes to escape from the trapping of the first (green-emitting) QW and enter the second and third (blue-emitting) QWs. Therefore, with a higher junction temperature, the blue emission becomes stronger relative to the green emission in our two-wavelength flip-chip $\mu$-LEDs. Among the three $\mu$-LEDs, sample D of the largest mesa area is supposed to have the highest junction temperature, followed by sample $\mathrm{C}$. The results of the saturation current density in Fig. 3 confirm this hypothesis. The observation of the most significant saturation behavior of sample D is consistent with the hypothesis. Meanwhile, because a higher junction temperature results in a higher conductivity in a semiconductor, the hypothesis of the highest junction temperature in sample D is in agreement with its highest conductivity, as shown in Fig. 4. To interpret the observed trend, we can first assume that the operation heat generation per

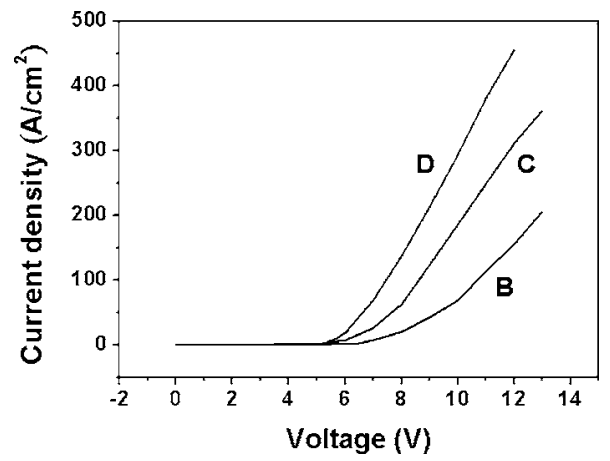

FIG. 4. Current density vs voltage curves of the three flip-chip $\mu$-LEDs with different mesa areas. unit mesa area is the same among the three $\mu$-LEDs. However, with the largest mesa area in sample $\mathrm{D}$, its ratio of the sidewall surface area over the active volume is the smallest, leading to the least effective sidewall heat radiation and light extraction. Because the heat radiation area on the $p$-type surface is the same as the operation heat generation area in each sample, the differences in heat radiation and light extraction between the three $\mu$-LEDs are determined by the mesa sidewall factor. Hence, the thermal effect and hence the junction temperature in sample D of the largest mesa area are the highest.

In summary, we have fabricated blue/green twowavelength, InGaN/GaN QW, flip-chip $\mu$-LEDs of different mesa sizes by stacking QWs of different indium contents. It was found that the blue-over-green contrast ratio of such a $\mu$-LED increased with the mesa size. The relatively stronger blue intensity in a device of a larger mesa area was due to its higher operation junction temperature for easier hole trapping of the deeper QWs.

This research was supported by National Science Council of Taiwan, Republic of China, under Grant No. NSC 942210-M-002-006 and by U.S. Air Force of Scientific Research under Contract No. AOARD-05-4085

${ }^{1}$ M. Yamada, Y. Narukawa, and T. Mukai, Jpn. J. Appl. Phys., Part 2 41, L246 (2002).

${ }^{2}$ B. Damilano, N. Grandjean, C. Pernot, and J. Massier, Jpn. J. Appl. Phys., Part 2 40, L918 (2001).

${ }^{3}$ D. Xiao, K. W. Kim, S. M. Bedair, and J. M. Zavada, Appl. Phys. Lett. 84, 672 (2004).

${ }^{4}$ J. Wu, W. Walukiewicz, K. M. Yu, J. W. Ager III, E. E. Haller, H. Lu, W. J. Schaff, Y. Saito, and Y. Nanishi., Appl. Phys. Lett. 80, 3967 (2002).

${ }^{5}$ I. Ozden, E. Makarona, A. V. Nurmikko, T. Takeuchi, and M. Krames, Appl. Phys. Lett. 79, 2532 (2001).

${ }^{6}$ Y. D. Qi, H. Liang, W. Tang, Z. D. Lu, and K. M. Lau, J. Cryst. Growth 272, 333 (2004).

${ }^{7}$ M. Hao, H. Ishikawa, T. Egawa, C. L. Shao, and T. Jimbo, Appl. Phys. Lett. 82, 4702 (2003).

${ }^{8}$ S. Fujita, M. Funato, D. C. Park, Y. Ikenaga, and S. Fujita, MRS Internet J. Nitride Semicond. Res. 4S1, G6.31 (1999).

${ }^{9}$ H. Nakayama, P. Hacke, and M. R. H. Khan, Jpn. J. Appl. Phys., Part 2 35, L282 (1996).

${ }^{10}$ K. S. Kim, M. G. Cheong, C. H. Hong, G. M. Yang, K. Y. Lim, E. K. Suh, and H. J. Lee, Appl. Phys. Lett. 76, 1149 (2000).

${ }^{11}$ H. W. Choi, M. D. Dawson, P. R. Edwards, and R. W. Martin, Appl. Phys. Lett. 83, 4483 (2003).

${ }^{12}$ H. W. Choi, C. W. Jeon, M. D. Dawson, P. R. Edwards, and R. W. Martin, IEEE Photonics Technol. Lett. 15, 510 (2003).

${ }^{13}$ S. X. Jin, J. Shakya, J. Y. Lin, and H. X. Jiang, Appl. Phys. Lett. 78, 3532 (2001).

${ }^{14}$ S. X. Jin, J. Li, J. Z. Li, J. Y. Lin, and H. X. Jiang, Appl. Phys. Lett. 76, 631 (2000).

${ }^{15}$ R. A. Mair, K. C. Zeng, J. Y. Lin, H. X. Jiang, B. Zhang, L. Dai, H. Tang, A. Botchkarev, W. Kim, and H. Morkoç, Appl. Phys. Lett. 71, 2898 (1997).

${ }^{16}$ S. M. Ting, J. C. Ramer, D. I. Florescu, V. N. Merai, B. E. Albert, A. Parekh, D. S. Lee, D. V. Christini, L. Liu, and E. A. Armour, J. Appl. Phys. 94, 1461 (2003). 